\title{
Emotional Face Processing in Pediatric Bipolar Disorder: Evidence for Functional Impairments in the Fusiform Gyrus
}

\author{
Susan B. Perlman, Ph.D., Jay C. Fournier, Ph.D., Genna Bebko, Ph.D., Michele A. Bertocci, \\ Ph.D., Amanda K. Hinze, M.S., Lisa Bonar, B.S., Jorge R. C. Almeida, M.D., Ph.D., Amelia \\ Versace, M.D., Claudiu Schirda, Ph.D., Michael Travis, M.D., Mary Kay Gill, R.N., M.S.N., \\ Christine Demeter, M.A., Vaibhav A. Diwadkar, Ph.D., Jeffrey L. Sunshine, M.D., Ph.D., \\ Scott K. Holland, Ph.D., Robert. A. Kowatch, M.D., Ph.D., Boris Birmaher, M.D., David \\ Axelson, M.D., Sarah M. Horwitz, Ph.D., L. Eugene Arnold, M. D., M.Ed., Mary. A Fristad, \\ Ph.D, A.B.P.P., Eric A. Youngstrom, Ph.D., Robert L. Findling, M.D, M.B.A., and Mary L. \\ Phillips, M.D., M.D. \\ Drs. Perlman, Fournier, Bebko, Bertocci, Almeida, Versace, Schirda, Travis, Birmaher, Axelson, \\ and Phillips, Ms. Hinze, Ms. Bonar, and Ms. Gill are with the Western Psychiatric Institute and \\ Clinic, University of Pittsburgh Medical Center, University of Pittsburgh. Dr. Phillips is also with \\ Cardiff University. Ms. Demeter and Drs. Sunshine and Findling are with the University Hospitals \\ Case Medical Center/Case Western Reserve University. Dr. Findling is also with Johns Hopkins \\ University. Dr. Diwadkar is with Wayne State University. Dr. Holland is with the Cincinnati \\ Children's Hospital Medical Center, University of Cincinnati. Dr. Kowatch is with the Research \\ Institute at Nationwide Children's Hospital. Dr. Horowitz is with New York University School of \\ Medicine. Drs. Arnold and Fristad are with Ohio State University. Dr. Youngstrom is with the \\ University of North Carolina at Chapel Hill.
}

\section{Abstract}

Objective-Pediatric bipolar disorder involves poor social functioning, but the neural mechanisms underlying these deficits are not well understood. Previous neuroimaging studies have found deficits in emotional face processing localized to emotional brain regions. However, few studies have examined dysfunction in other regions of the face processing circuit. This study assessed hypoactivation in key face processing regions of the brain in pediatric bipolar disorder.

Method-Youth with a bipolar spectrum diagnosis $(n=20)$ were matched to a nonbipolar clinical group $(n=20)$, with similar demographics and comorbid diagnoses, and a healthy control group $(n=20)$. Youth participated in a functional magnetic resonance imaging (fMRI) scanning which employed a task-irrelevant emotion processing design in which processing of facial emotions was not germane to task performance.

(C) 2013 American Academy of Child \& Adolescent Psychiatry. Published by Elsevier Inc. All rights reserved.

Correspondence to Susan B. Perlman, Ph.D., Western Psychiatric Institute and Clinic, Loeffler Building, Room 121, 121 Meyran Avenue, Pittsburgh, PA 15213; perlmansb2@upmc.edu.

Publisher's Disclaimer: This is a PDF file of an unedited manuscript that has been accepted for publication. As a service to our customers we are providing this early version of the manuscript. The manuscript will undergo copyediting, typesetting, and review of the resulting proof before it is published in its final citable form. Please note that during the production process errors may be discovered which could affect the content, and all legal disclaimers that apply to the journal pertain.

Supplemental material cited in this article is available online.

Disclosure: Drs. Perlman, Fournier, Bebko, Bertocci, Almeida, Versace, Schirda, Travis, Diwadkar, Holland, Axelson, Horwitz, and Phillips, Ms. Hinze, Ms. Bonar, Ms. Gill, Ms. Demeter report no biomedical financial interests or potential conflicts of interest. 
Results-Hypoactivation, isolated to the fusiform gyrus, was found when viewing animated, emerging facial expressions of happiness, sadness, fearfulness, and especially anger in pediatric bipolar participants relative to matched clinical and healthy control groups.

Conclusions-The results of the study imply that differences exist in visual regions of the brain's face processing system and are not solely isolated to emotional brain regions, such as the amygdala. Findings are discussed in relation to facial emotion recognition and fusiform gyrus deficits previously reported in the autism literature. Behavioral interventions targeting attention to facial stimuli might be explored as possible treatments for bipolar disorder in youth.

\section{Keywords}

emotion; face processing; functional magnetic resonance imaging (fMRI); fusiform; gyrus; pediatric bipolar disorder

\section{Introduction}

Pediatric bipolar spectrum disorders (BPSD), including bipolar I and II disorders, cyclothymic disorders, and bipolar disorder not otherwise specified (NOS), are debilitating illnesses affecting approximately $2 \%$ of the child and adolescent population ${ }^{1}$. In addition to the well documented clinical impairments found in children suffering from BPSD ${ }^{2}$, Geller $e t$ $a l .{ }^{3}$ reported significant psychosocial deficits. They found that the majority of children with BPSD had few friends, poor social skills, and hostile relationships with parents and siblings. While it is difficult to disentangle whether these psychosocial deficits are a cause or an effect of the disorder, research into basic social processes, and the accompanying neural deficits related to these processes, can help inform therapeutic treatments for children with BPSD.

Several studies of pediatric BPSD ${ }^{4-10}$ have focused on emotional face processing due to its associations with social impairment ${ }^{11}$. Youth with BPSD have lower accuracy in identifying emotional facial expressions compared to their healthy counterparts or children with other forms of psychopathology(e.g. ADHD, ODD, anxiety $)^{8}$. They also have impairments in memory of faces ${ }^{12}$ and are more likely to misinterpret neutral faces as threatening ${ }^{5}$. Further, this effect has been found in those youth at familial risk of BPSD ${ }^{13}$. Functional Magnetic Resonance Imaging(fMRI) studies have also examined impairment in emotional face processing, mostly focusing on dysfunction in the amygdala, an important emotional brain region in the face processing circuit ${ }^{5,6}$. Rich et al. found amygdala hyperactivation, compared to control participants, when rating perceived fear of an emotional face ${ }^{5}$ and reduced functional connectivity to other regions in the face processing circuit ${ }^{6}$.

Although there is a wealth of literature indicating amygdala dysfunction during judgment of emotional faces, few, if any, studies have focused on other parts of the emotional face processing system in pediatric BPSD. One critical component of the brain's face processing circuit is the "fusiform face area," a region localized to the lateral fusiform gyri (FG) that is specialized for face perception ${ }^{14,15}$. Limited evidence indicates that there may also be impairment in this area in pediatric BPSD. While neural activity in the FG was not examined directly, Rich et al. found decreased connectivity between the hyperactivated amygdala and this region on an emotional face judgment task in children with BPSD compared to their healthy counterparts ${ }^{6}$. This may indicate abnormal functioning in the FG itself or simply inefficient communication within the face processing circuit (e.g., hyperactivation of the amygdala as a compensatory attempt to communicate with the FG through impaired connections). Adleman et al. also found that faces that were remembered vs. forgotten in youth with BPSD were encoded differently in the FG compared to healthy 
subjects $^{12}$. Studies of adult patients with BPSD have examined structural changes in this region with inconclusive results. Adler et al. showed increased gray matter volume in the $\mathrm{FG}^{16}$ while Moorhead et al. reported a decline in gray matter density, compared to control participants, over a 4 -year period ${ }^{17}$.

A second brain region of importance in the face processing circuit is the superior temporal sulcus (STS), which is noted for its role in the perception of biological motion and social action interpretation ${ }^{18}$. The STS is particularly sensitive to the eye-gaze ${ }^{19,20}$, head movement $^{21}$, and mouth motion ${ }^{22}$. Very little research has focused on the STS in children with BPSD, likely due to the lack of evidence of impaired biological motion processing in this group. Pavuluri et al. ${ }^{23}$ did, however, find that pediatric BPSD patients showed elevated activation in the STS, in comparison to healthy children, while viewing angry faces.

The current study was designed to test the possibility of impairment in the entirety of the face processing system in youth with BPSD, which could potentially underlie deficits in emotional face judgment and social interaction ${ }^{3,8}$. Given the previous literature indicating poor performance in emotional face judgment tasks, the instructions for the task used in the current study did not focus on emotion judgment, potentially enhancing our sensitivity to examine differences outside limbic regions. This allowed us to eliminate the possibility that findings might be due to higher order differences in cognitive functioning rather than discrete visual processing of facial stimuli. Further, given evidence for impairments in FG and STS activity in other forms of psychopathology ${ }^{24-26}$, we collected data from an additional group of children who were matched on demographic characteristics and comorbid psychopathology to the BPSD group(e.g., anxiety, disruptive behavior, attentiondeficit/hyperactivity disorder [ADHD]), but who did not have BPSD, as well as a nonclinical healthy control group.

\section{Method}

All methods for recruitment and participant testing were approved by the institutional review boards (IRBs) of all participating universities.

\section{Participants}

Forty youth (9.89-16.88 years; $\mathrm{M}=13.61, \mathrm{SD}=1.95)$ who had been recruited into the original Longitudinal Assessment of Manic Symptoms Study (LAMS1) participated in the second phase of this project (LAMS2). LAMS1 was a multi-site study in which youth, ages 6-12, were recruited as they sought care for a variety of diagnoses in nine mental health outpatient clinics associated with the 4 collaborating universities. The original goal of LAMS1 was to employ longitudinal clinical assessment to determine early risk for $\mathrm{BPSD}^{27,28}$. For the current study (LAMS2), beginning 5 years after the start of LAMS1, these youth returned to participate in neuroimaging procedures and clinical assessments (see below). From this sample, 20 youth who had obtained a bipolar spectrum diagnosis (BPSD) at or between entry into LAMS1 and start of LAMS2, and who had artifact-free neuroimaging data, were matched, to the best of our ability, to 20 clinic youth without a bipolar spectrum diagnosis (Non-BPSD) on demographic characteristics (age, sex, IQ, socioeconomic status [SES]) and comorbid diagnoses. The Non-BPSD clinical group was quite heterogeneous in diagnosis but did not statistically differ from the BPSD group in categories of comorbid diagnosis (see Table 1). The BPSD group comprised 11 subjects with bipolar I disorder, 6 subjects diagnosed with bipolar NOS, and 3 subjects with cyclothymic disorder. No subjects in this sample had been diagnosed with bipolar II disorder. The amount of time between intake into the LAMS1 study and participation in the LAMS2 scan procedure ranged between 2.47 and 5.97 years (mean $=4.42$ years, $S D=0.83$ years). An additional, newly recruited, sample of 20 mentally and physically healthy participants (Control) without personal or family history of 
mood disorders in first degree, and without family history of BPSD in second degree, relatives were also included in the neuroimaging procedures. These participants were matched on demographic variables to the BPSD and Non-BPSD participants (see Table 1).

Participants were recruited from 3 of the LAMS sites: Case Western Reserve University ( $\mathrm{n}=24,9$ BPSD, 6 Non-BPSD, 9 Control); Cincinnati Children's Hospital ( $\mathrm{n}=15$, 7 BPSD, 7 Non-BPSD, 1 Control); and University of Pittsburgh Medical Center/Western Psychiatric Institute and Clinic ( $\mathrm{n}=21,4$ BPSD, 7 Non-BPSD, 10 Control). Parents/guardians provided written informed consent, and youth provided written informed assent prior to study participation. Participants received monetary compensation and a framed picture of their structural neuroimaging $\operatorname{scan}^{29}$.

Exclusion criteria included severe systemic medical illnesses, neurological disorders, history of head trauma with loss of consciousness, use of medications that may produce central nervous system (CNS) effects (e.g., steroids), IQ<70 (assessed by the Wechsler Abbreviated Scale of Intelligence $)^{30}$, positive urine drug and/or salivary alcohol screen on the day of the scan, alcohol or substance abuse in the past 3 months (determined by the Schedule for Affective Disorders and Schizophrenia for School Age Children, Present and Life Version; K-SADS-PL-W ${ }^{31}$ ), visual disturbance (less than 20/40 Snellen visual acuity), being unable to complete questionnaires in English, parent-reported history of physical/sexual abuse, autism spectrum disorders or developmental delays, posttraumatic stress disorder, and taking more than 3 different psychotropic medications. Additional exclusion criteria for scanning included pregnancy, claustrophobia, or metal objects in the body. LAMS youth were permitted to use prescribed medication(s) before scanning, given ethical problems with stopping medication for research purposes.

\section{Symptom Assessment}

Diagnostic assessments of the LAMS participants were performed annually by interviewing the caregiver and child using the K-SADS-PL-W. In addition, the mood modules of the KSADS-PL-W interview were performed semianually ${ }^{27,28}$. The number of months between the nearest K-SADS-PL-W assessment and scan date ranged between 3.9 months before the scan to 4.5 months after the scan. Based on this interview, participants were given a yes/no score for a lifetime history of each of 5 diagnostic categories (BPSD, depression, anxiety, ADHD, and disruptive behavior disorders [i.e., conduct disorder or oppositional defiant disorder]). No participant was given a lifetime history diagnosis of a substance use disorder (see Table 1). BPSD and Non-BPSD participants were matched on all diagnoses other than BPSD and on the demographic variables mentioned above.

\section{Paradigm}

Participants completed a 13-minute emotional dynamic faces task ${ }^{32}$ during fMRI scanning. Participants were asked to use one of three fingers to press a button indicating the color of a semitransparent foreground color flash (orange, blue or yellow) that appeared during the mid $200 \mathrm{~ms}-650 \mathrm{~ms}$ of a 1 -second presentation of a dynamically changing background face (neutral to emotional; see Figure 1). These emotional faces occupied the majority of the screen (approximately 23 visual degrees) and were interleaved with a central crosshair that was jittered to remain on the screen for 2-3 seconds. Faces from the NimStim stimulus set ${ }^{33}$ were morphed in 5\% increments, from neutral ( $0 \%$ emotion) to $100 \%$ emotion for 4 emotions: happy, sad, angry, and fearful. Participants were told to ignore the face as it was not relevant to their task. Morphed faces were made into $1 \mathrm{~s}$ movies progressing from $0 \%$ to $100 \%$ emotion. In control trials, movies comprised a simple shape (dark oval) superimposed on a light-grey oval, which was subsequently morphed into a larger shape, approximating the movement shown by the morphed faces. There were 3 blocks for each of the above 4 
types of emotion trial, with 12 stimuli per block (42 second blocks), and 12 control blocks, with 6 stimuli per block ( 21 second blocks). Control blocks were half the duration of emotional blocks in order to minimize the time difference between baseline and conditions for individual emotion contrasts and lessen the overall time burden on young participants. Emotional blocks were presented in a pseudorandomized order so that no two blocks of any condition were presented sequentially. Control blocks were interleaved with emotional blocks. The task was slightly modified from previously published adult versions for use with children ${ }^{32,34}$. A graphical reminder of which color was assigned to each button was visible on the right side of the screen for the duration of the experiment.

\section{Data Acquisition}

At the Pittsburgh site neuroimaging data were collected on a 3.0 Tesla Siemens Trio, at the Cleveland site neuroimaging data were collected with a 3.0 Tesla Siemens Verio, and at the Cincinnati site neuroimaging data were collected with a Phillips Achieva 3.0 Tesla X-series scanner. Structural 3D axial MPRAGE/PAR-REC images were acquired in the same session (repetition time $[\mathrm{TR}] /$ time to echo $[\mathrm{TE}]=2200 / 3.29 \mathrm{~ms}$, Flip angle $9^{\circ}$, field of view [FOV]: $256 \times 192 \mathrm{~mm}^{2}$, Slice thickness: $1 \mathrm{~mm}$, Matrix: 256×256, 192 continuous slices). Bloodoxygen-level-dependent (BOLD) images were then acquired with a gradient echo-planar imaging (EPI) sequence during approximately 13 minutes (386 successive brain volumes) covering 39 axial slices $\left(3.2 \mathrm{~mm}\right.$ thick, TR/TE $=2000 / 28 \mathrm{~ms} / \mathrm{ms}, \mathrm{FOV}=205 \times 205 \mathrm{~mm}^{2}$, matrix $=64 \times 64$; Flip angle $90^{\circ}$ ). Imaging parameters were adjusted identically on all 3 scanners.

Previous studies ${ }^{35,36}$ indicate the feasibility of combining structural and functional neuroimaging data from different sites but emphasize the necessity of measuring, and controlling for intersite differences in scanner signal-to-noise ratio (SNR). To ensure intrasite reliability among scanners at our 3 study sites and to allow combination of neuroimaging data across sites, we used the recommended standards published by the Biomedical Informatics Research Network (BIRN; http://www.nbirn.net) for data acquisition and information sharing by measuring scanner SNR monthly with BIRNrecommended phantoms at each site. Additionally, we explored the effects of site and monthly SNR in our final analyses (see below).

\section{Data Analysis}

Data were preprocessed and analyzed using BrainVoyager QX 2.4 (Brain Innovation, Maastricht, The Netherlands). Preprocessing included slice time correction (cubic spline interpolation), alignment of slice (cubic spline interpolation to the first nondiscarded scan time), 3-dimensional motion correction (trilinear interpolation), spatial smoothing $(6 \mathrm{~mm}$ Gaussian kernel), linear trend removal, and temporal high-pass filtering (fast-Fourier transform based with a cutoff of 3 cycles/time course). The functional data sets were coregistered to the Talairach-transformed ${ }^{37} \mathrm{~T} 1$-weighted anatomical image series to create a 4-dimensional data representation.

Z-transformed participant movement was entered as a covariate of no interest at the individual participant level. No participants included in this analysis moved more than $3 \mathrm{~mm}$ from their starting head position, neither from movement spikes nor from slow drift, during the course of data collection. Groups did not significantly differ in mean frame displacement $(F[2,57]=0.49 p=0.61)$.

Although we were primarily interested in the face processing system, our primary analysis strategy employed a whole-brain, conservative approach in order to allow for unexpected findings in other brain regions. A secondary analysis restricted our regions of interest to 
those of the face processing system (Amygdala, FG, STS) using a more liberal threshold. For both analyses, a multi-participant statistical analysis was performed by multiple linear regression of the time course of the BOLD response in each voxel across the whole brain. Regressors were generated to represent the design matrix of the experiment and a general linear model was computed to fit these regressors to each participant's z-normalized volume time courses. Model predictors were defined by convolving an ideal boxcar response with a gamma-function model of the hemodynamic response ${ }^{38}$. Boxcar values were equal to 1 during the emotion morph blocks and 0 during shape morph blocks.

First, we computed a 3 (group: BPSD, Non-BPSD, Control) $\times 4$ (condition: happy, sad, anger, fear) analysis of variance (ANOVA), with shapes as the baseline condition, to examine the main effects of condition and group and the group $\times$ condition interaction across the whole brain. Activation maps were visualized on a Talairach-transformed template brain, and displayed at a resolution of $1 \mathrm{~mm}^{3}$, and all $p$-values for all analyses were subjected to a whole-brain threshold of $p<.001$ and a $203 \mathrm{~mm}^{3}$ voxel extent, which is slightly more conservative than the $p<.005$ and a 10 voxel extent threshold that has been proposed in order to balance Type I and Type II error ${ }^{39}$. Beta values were extracted from regions displaying the effects of interest and plotted to examine differences between conditions and groups. Finally, a replication of the analysis described above was conducted using a region of interest mask of the face processing system (bilateral amygdala, FG, and STS). Maps for this analysis were subjected to a more liberal threshold of $p<.001$, with no voxel extent threshold.

In order to examine the effects of behavioral, demographic, and clinical variables on our neuroimaging effects, we computed the contribution of these variables to our findings using SPSS version 20 (IBM Software). Across all groups, relationships were examined between accuracy and reaction time during color labeling in emotion blocks, as well as demographic variables, and beta values for extracted activation differences from baseline. In both patient groups, relationships were examined between comorbid diagnoses and medication usage and these beta values. A problem for all neuroimaging studies of BPSD is the potential confounding effect of psychotropic medication, as it is difficult to recruit medication-free participants into such studies ${ }^{40}$. Thus, variables representing the taking versus not taking of each psychotropic medication class (antipsychotic, antidepressant, mood stabilizer, and stimulant) were examined. All analyses were computed by univariate ANCOVA with extracted BOLD signal as the dependent variable, group (BPSD, non-BPSD, control) as the independent variable, and the variable of interest as a covariate.

\section{Results}

See Supplement 1 and Figures S1 and S2, available online, for a discussion of data combination across multiple sites.

\section{Task Performance}

Color labeling accuracy and reaction times for emotion blocks were calculated based on individual participant task performance for each of the 5 conditions (fearful, angry, sad, happy, and shapes). Overall, color labeling accuracy was $76 \%, 80 \%$, and $92 \%$ for BPSD, Non-BPSD, and Control, respectively. We computed a repeated measures ANOVA for accuracy and reaction time with 3 groups (BPSD, Non-BPSD, Control) $\times 5$ conditions (happy, sad, anger, fear, shapes). For task accuracy, we found a main effect of group ( $F$ $[2,56]=4.68, p=.013)$ and a main effect of condition $(F[4,224]=4.35, p=.002)$, but no group $\times$ condition interaction. Post-hoc tests of group differences, with a least significant difference (LSD) correction, revealed decreased accuracy of both the BPSD group (mean difference=. $16, p=.005$ ) and Non-BPSD group (mean difference=.12, $p=.03$ ) compared to the control 
group, but the accuracy difference between the two patient groups was not significant. Pairwise comparisons for each condition, with a LSD correction, revealed decreased accuracy for the happy condition compared to the sad (mean difference $=-.03, p=.01$ ) and angry (mean difference $=-.04, p=.001$ ) conditions and an increased accuracy for the anger condition compared to the fear condition (mean difference $=-.03, p=.01$ ), but not between any other conditions. Although there was no significant group $\times$ condition interaction for accuracy, we note that scores differed significantly between the BPSD and Control groups for all conditions (smallest mean difference $=.14$, all $p \mathrm{~s} \leq 02$ ) and did not significantly differ between patient groups on any conditions (largest mean difference=.06, all $p s \searrow 0.28$; see Figure 2).

For reaction time, we found a main effect of condition $(F[4,224]=4.892, p=.001)$, but no main effect for group or a group $\times$ condition interaction. Pairwise comparisons for each condition, with a LSD correction, revealed increased reaction time for the sad condition compared to the fearful (mean difference $=40.42, p=.001$ ) and shapes (mean

difference $=43.56, p<.001$ ) conditions and an increased reaction time for the anger, compared to the shape conditions (mean difference $=25.92, p=.005$ ), but not between any other conditions. Results did not implicate a speed-accuracy trade off (i.e. significant positive correlation between speed and accuracy) in the BPSD and Control groups. Overall speed and accuracy were positively correlated for the Non-BPSD group $(r[18]=.55, p=.012)$.

\section{Neural Activity}

The 3 (Group: BPSD, Non-BPSD, Control) $\times 4$ (Condition: happy, sad, angry, fearful) ANOVA revealed a main effect of group in the left inferior frontal gyrus $(F[2,57] \geq 7.82, p<$. 001; see Table 2 for specific cluster size, statistical value, and location information). Contrasts of the average baseline-corrected neural activity beta values extracted from the whole of the active cluster, computed using SPSS v20, revealed significant mean differences between all groups. The Control group had the highest change from baseline (mean=.16, $\mathrm{SE}=.03$ ), followed by the Non-BPSD group (mean=.07, $\mathrm{SE}=.03$ ), followed by the BPSD group (mean=-.05, $\mathrm{SE}=.03$ ), whose activity decreased from baseline. There was also a main effect of condition $(F[3,171] \geq 5.67, p<.001)$, located in the bilateral superior frontal gyrus. Here, fear (mean=.10, $\mathrm{SE}=.04)$ and happy (mean=.05, $\mathrm{SE}=.03$ ) were increased from baseline, though not significantly different from each other, while sad (mean=-.07, SE= .04) and anger (mean=-.17, $\mathrm{SE}=.04$ ) decreased from baseline and were each significantly different from all other conditions.

Finally, a significant group $\times$ condition interaction, revealed a single cluster of activation located in the left FG $(F[6,171] \geq 3.95, p<.001$; see Figure 3$)$. This cluster was located in the vicinity of the fusiform face area based on the work of Kanwisher et al. ${ }^{14}$ We created a region of interest based on a $3 \mathrm{~mm}$ sphere centered at voxel $\mathrm{x}=-35, \mathrm{y}=-63, \mathrm{z}=-10$, which was reported by Kanwisher et al. as the center of the left fusiform face area. Beta values for baseline corrected neural activity levels were then extracted from this region of interest for the group $\times$ condition interaction in order to further explore the contributions of each individual condition and group to the interaction. The results of a one-way ANOVA for each condition revealed significant group differences only for the angry condition $(F[2,57]=18.12, p<.001)$ in this area of the FG. Post-hoc LSD contrasts revealed that the BPSD group had significantly lower activity levels within the left FG region compared to the Controls $(p<.001)$ the Non-BPSD group ( $p<.001$; see Figure 3$)$. There were nonsignificant trends for the one-way ANOVAS for the $\operatorname{sad}(F[2,57]=2.99, p=.058)$ and fear $(F[2,57]=2.80, p=.069)$ conditions.

In order to increase our power to find effects of interest solely within the face processing system, we computed the above analysis using a bilateral mask of the amygdala, FG, and 
STS with a liberal overall threshold of $p<.001$, uncorrected. This ANOVA revealed a main effect of group in the left FG $(F[2,57] \geq 7.82, p<.001$; see Table 2 for specific cluster size and location information). Contrasts of baseline corrected neural activity beta values extracted from this region revealed significant mean differences between the BPSD and both other groups but not between the Control and Non-BPSD group. The Non-BPSD group had the highest change from baseline (mean $=.36, \mathrm{SE}=.06$ ), followed by the Control group (mean=.30, $\mathrm{SE}=.06$ ), followed by the BPSD group (mean=-.01, $\mathrm{SE}=.06)$. There was also a main effect of condition $(F[3,171] \geq 5.67, p<.001)$, located in the right STS. Here, anger (mean=.25, SE= .04), fear (mean=.24, $\mathrm{SE}=.05)$ and sad (mean=.19, $\mathrm{SE}=.04)$ did not significantly differ from each other but were all 3 higher than happy (mean=.08, SE= .04).

Finally, a significant group $\times$ condition interaction, revealed a cluster of activation located in the left FG $(F[6,171] \geq 3.95, p<.001)$, overlapping with the interaction effect found at the whole-brain level. No effect was found in the right or left amygdala.

\section{Relationships Between Demographic Variables, Task Performance, Clinical Variables}

To examine the potential effects of demographic variables, task performance, and clinical variables on the between-group differences found within the FG, the baseline corrected extracted activation betas, taken from the region of the FG in which the group $\times$ condition interaction was significant (see Figure 2), were used as the dependent variable in a series univariate analyses of covariance (ANCOVAs). Each ANCOVA model had 2 independent variables, diagnostic group and the covariate of interest. Here, we used the FG activation for only the anger condition because it was noted to have the largest difference between groups. This focused analysis also allowed us to reduce the likelihood of committing a Type 1 error.

The demographic variables, age, sex, SES, and IQ did not have an independent effect on FG activation to anger (largest $F[1,56]=2.13, p=.15$ ). In each model, the effect of diagnostic group remained significant even including the covariate in the model (all $F \mathrm{~s}[2,56] \geq 15.29$, all $p \mathrm{~s}<.001)$. For behavioral variables, task accuracy $(F[1,56]=.68, p=.41)$ and reaction time $(F[1,56]=1.96, p=.17)$ for the anger condition did not have independent effects on FG activation. The effect of diagnostic group remained significant when controlling for task accuracy $(F[2,56]=16.85, p<.001)$ and reaction time $(F[2,56]=18.54, p<.001)$.

For clinical variables, we examined only the BPSD and Non-BPSD groups. Lifetime history of comorbid anxiety disorders, ADHD, or disruptive behavior disorders did not have an independent effect on anger FG activation (all $F \mathrm{~s}[1,37] \leq .57$, all $p \mathrm{~s} \geq 22$ ), and the effect of diagnostic group remained significant in each model (all $F \mathrm{~s}[1,37] \geq 31.00$, all $p \mathrm{~s}<.001$ ). Finally, when examining medication effects, use of neither antidepressants, antipsychotics, mood stabilizers, nor stimulants (taking vs. not taking) had an independent effect on anger FG activation (all $F \mathrm{~s}[1,37] \unlhd .55$, all $p \mathrm{~s} \geq 22$ ), and the effect of diagnostic group remained significant in each case (all $F_{\mathrm{s}}[1,37] \geq 27.48$, all $p \mathrm{~s}<.001$ ).

\section{Data Combination from Multiple Scan Sites}

As described earlier, scanner SNR was collected monthly with a BIRN phantom and linked to each participant's scan data. Following the same univariate ANCOVA procedure utilized to examine the effects of demographic, task performance, and clinical variables, we examined the effect of SNR on average FG activation across emotions. In this model, diagnostic group, scanner SNR, and scan site were entered as independent variables. Neither SNR nor scan site had an independent effect on average FG activation (all $F \mathrm{~s}[1,56] \leq 647$, all $p \mathrm{~s} \geq 43$ ), and the effect of diagnostic group on average FG activation remained significant (all $F \mathrm{~s}[2,56] \geq 4.99$, all $p \mathrm{~s} \leq 01$ ). For additional analyses relating to multisite data compilation, see supplemental materials. 


\section{Discussion}

Using a dynamic face processing task, we found evidence for decreased activation of the left FG in youth with BPSD relative to both a clinical group matched for demographic characteristics and comorbid psychopathology and a demographically matched healthy control group. This effect was present in all emotion conditions, but was most apparent for the anger condition.

Examining all areas of the brain, we found that it was only the FG ("fusiform face area") region that showed significant interaction between groups and conditions. This region is widely noted as a region involved in not only face processing, but key components of social communication $^{41}$ such as eye contact ${ }^{42}$, facial identity recognition ${ }^{43}$, and mouth movement ${ }^{44}$. Although this region has rarely been studied directly in pediatric BPSD, inferences can be drawn from widely replicated results of hypoactivation and social impairment in the autism literature ${ }^{45-47}$. Dalton et al. ${ }^{46}$, for example, found that visual fixation upon the eyes of a face was positively correlated with FG activation while Kleinhans et al. ${ }^{47}$ found that amygdala to FG functional connectivity was negatively correlated with social impairments as diagnosed through clinical interview. Future studies may, therefore, show that similar impairments in social functioning may be linked to FG hypoactivation in pediatric bipolar disorder. Indeed, in the current study we found FG hypoactivation in our BPSD group, but not in our group matched for comorbid diagnosis, whose fusiform activation did not differ from that of a healthy control group. This study provides preliminary evidence that dysfunction in emotion processing occurs not only in emotional regions of the brain, which might be expected given the affective challenges of this mood disorder, but also in basic visual processing of faces. Additionally, we found a main effect of group in the inferior frontal gyrus, which was highest for the Control group, possibly indicating increased regulation of affect in response to emotionally evocative stimuli.

Although participants were asked to perform a task that did not require explicit attention to emotional faces, we found that FG activation varied according to the type of emotional distracter in the BPSD group. Specifically, the differences in activation between the BPSD group and the Non-BPSD group were largest for the angry face condition and smaller, though still statistically significant, for the sad face condition. Not only is anger an emotion that is often present in the clinical symptoms of pediatric BPSD ${ }^{2,48}$, but also previous studies have pointed to deficits in anger perception in BPSD. In a pediatric BPSD sample Guyer $e t a l .{ }^{8}$ reported the most errors in identifying angry faces compared to other emotions, and Rich et al. ${ }^{5}$ reported that neutral faces were perceived as more "hostile" by youth with BPSD compared to control participants. Neuroimaging studies also have found increased activation in the amygdala and prefrontal cortex during passive viewing of angry faces in comparison to control participants ${ }^{49}$. Our results indicate decreased visual processing of angry faces in participants with BPSD. This may underlie social impairments often seen in these youth, who may not effectively detect or may misinterpret the facial cues present in angry friends or family members.

Many previous studies indicate that there is an overall right greater than left laterality in FG face-selective activity ${ }^{14,50-52}$, however, our results were localized to the left hemisphere. Our findings were mostly driven by the angry face condition. Previous research aimed at emotional brain asymmetry has indicated lateralized brain activity in the right hemisphere for withdrawal emotions (e.g., sadness) and lateralized brain activity in the left hemisphere for approach emotions (e.g., anger) ${ }^{53}$. For example, in a study of electroencephalography (EEG) asymmetry, Harmon-Jones ${ }^{54}$ found that in adults who self-reported high trait anger, greater relative left frontal cortical activity was observed to anger-invoking pictures, but not 
to other emotional pictures. Although measures of trait anger were not collected in this sample, the FG asymmetry results observed may be related to differences in emotional perception of this approach emotion between groups.

Although the findings of this study are promising, some limitations must be noted. Nearly all clinical participants in this study were medicated and, although covariate analyses indicate that medication usage did not affect the specific findings of our study, effects of medication on face perception systems are not well understood. However, previous studies have argued that medication is more likely to have a normalizing effect in clinical neuroimaging studies ${ }^{40}$, so we would not expect medication to introduce spurious differences. Second, limitations of the current work may be related to the fMRI study design. In contrast to previous studies of emotional face processing in pediatric bipolar disorder ${ }^{5,55}$, our analyses did not find differences in amygdala functioning when viewing emotional faces. This could be related to the task-irrelevant emotional nature of our design (requiring no emotional judgment). This could also be related to the lack of task conditions requiring differential focus on the emotional faces. Indeed, other research that has employed this technique has found modulation of the amygdala due to varying attentional demands placed on the stimulus ${ }^{56}$. Finally, the block design of our study, combined with the differing accuracy between subject groups on emotion and baseline conditions, can be seen as a limitation to our interpretation of study findings. The block design of the study did not allow us to analyze only correct trials, which were significantly fewer in the BPSD and Non-BPSD groups. As previous studies emphasized 46,57 , one must investigate the nature of the behavior involved in the task in order to disambiguate an effect of interest from other neural processes related to error rate (e.g., conflict monitoring ${ }^{58}$, executive function ${ }^{59}$ ). Additionally, the possible speed/accuracy trade-off observed in the Non-BPSD group points to possible behavioral differences among groups that is not easily noted with a block design. Thus, we encourage our results to be interpreted as an early phase investigation requiring further replication using event-related task design. Future studies should attempt the analysis of only correct trials when performance differences arise, which is a confound that is often unavoidable when investigating young patient groups. We note, however, that in our study, behavioral differences were only found between the patient and control groups and did not exist when comparing both patient groups. Thus any possible confound caused by behavioral performance cannot account for the observed differences between the BPSD and Non-BPSD children with regard to FG activity to Sad and Angry faces. Further mitigating the concern that performance confounds may be responsible for any of the results we observed, the Non-BPSD and healthy control groups did not differ from each other with regard to FG activity to Anger despite the fact that the Non-BPSD children were significantly less accurate than the controls during this task condition.

Results of this study suggest that FG deficits in facial processing are specific to pediatric BPSD rather than a common functional brain abnormality observed in general child psychopathology. Thus, it is possible that this patient group could benefit from therapeutic training designed to enhance FG functioning to facilitate positive social interaction. Similar interventions have been attempted, with mixed success, in studies of Autism Spectrum Disorders through eye-contact ${ }^{60}$ and facial expression recognition training ${ }^{61}$ as well as in psychosocial treatments for pediatric BPSD $^{50}$. Before such treatment development work can commence, however, future studies should consider employing the use of eye-tracking methodology during fMRI scanning to determine if the observed effect is due to visual avoidance of emotional faces or simply an effect of inefficiency in the brain's face processing circuit in pediatric BPSD. Additionally, studies enrolling young children at risk for BPSD (e.g., unaffected siblings) could examine longitudinally whether FG hypoactivation is a biological precursor of this disorder or the result of repeated difficulties in social interaction throughout the course of development. 


\section{Supplementary Material}

Refer to Web version on PubMed Central for supplementary material.

\section{Acknowledgments}

This research was supported by the National Institute of Mental Health (NIMH) grants 2R01 MH73953-06A1 (B.B., M.L.P.), 1K01 MH094467 (S.B.P.), 2R01 MH73816-06A1 (S.K.H., R.A.K.), 2R01 MH73967-06A1 (R.L.F.), and 2R01 MH73801-06A1 (M.A.F.).

Dr. Sunshine has received research support from Siemens Healthcare though not directly related to this study. Dr. Kowatch has served as a consultant to Forest Pharmaceutical, AstraZeneca, and the REACH Foundation. He has received research support from NIMH and is an editor for Current Psychiatry. Dr. Birmaher has received royalties from publications from Random House, Inc. (New Hope for Children and Teens With Bipolar Disorder) and Lippincott Williams and Wilkins (Treating Child and Adolescent Depression). He has received research funding from NIMH. Dr. Arnold has received research funding from Curemark, Forest, Eli Lilly and Co., and Shire; advisory board honoraria from Biomarin, Novartis, Noven, Roche, Seaside Therapeutics, and Shire; and travel support from Noven. He has served as a consultant to Tris Pharma. Dr. Fristad has received royalties from Guilford Press, American Psychiatric Publishing (APPI), and CFPSI. Dr. Youngstrom has served as a consultant to Lundbeck and has received grant support from the National Institutes of Health (NIH). Dr. Findling has received research support from, served as a consultant to, or served on the speakers' bureau for Alexza Pharmaceuticals, APPI, AstraZeneca, Bracket, Bristol-Myers Squibb, Cognition Group, Forest, GlaxoSmithKline, Guilford Press, Johns Hopkins University Press, Johnson and Johnson, KemPharm, Eli Lilly and Co., Lundbeck, Merck, NIH, Novartis, Noven, Otsuka, Pfizer, Physicians Postgraduate Press, Rhodes Pharmaceuticals, Roche, Sage, Seaside Pharmaceuticals, Shire, Stanley Medical Research Institute, Sunovion, Supernus Pharmaceuticals, Transcept Pharmaceuticals, Validus, and WebMD.

\section{References}

1. Van Meter AR, Moreira AL, Youngstrom EA. Meta-analysis of epidemiologic studies of pediatric bipolar disorder. J Clin Psychiatry. 2011 Sep; 72(9):1250-1256. [PubMed: 21672501]

2. Pavuluri MN, Birmaher B, Naylor MW. Pediatric bipolar disorder: a review of the past 10 years. J Am Acad Child Adolesc Psychiatry. 2005 Sep; 44(9):846-871. [PubMed: 16113615]

3. Geller B, Craney JL, Bolhofner K, Nickelsburg MJ, Williams M, Zimerman B. Two-year prospective follow-up of children with a prepubertal and early adolescent bipolar disorder phenotype. Am J Psychiatry. 2002 Jun; 159(6):927-933. [PubMed: 12042179]

4. McClure EB, Treland JE, Snow J, et al. Deficits in social cognition and response flexibility in pediatric bipolar disorder. Am J Psychiatry. 2005 Sep; 162(9):1644-1651. [PubMed: 16135623]

5. Rich BA, Vinton DT, Roberson-Nay R, et al. Limbic hyperactivation during processing of neutral facial expressions in children with bipolar disorder. Proc Natl Acad Sci U S A. 2006 Jun 6; 103(23): 8900-8905. [PubMed: 16735472]

6. Rich BA, Fromm SJ, Berghorst LH, et al. Neural connectivity in children with bipolar disorder: impairment in the face emotion processing circuit. J Child Psychol Psychiatry. 2008 Jan; 49(1):8896. [PubMed: 18181882]

7. Rich BA, Grimley ME, Schmajuk M, Blair KS, Blair RJ, Leibenluft E. Face emotion labeling deficits in children with bipolar disorder and severe mood dysregulation. Dev Psychopathol. 2008 Spring;20(2):529-546. [PubMed: 18423093]

8. Guyer AE, McClure EB, Adler AD, et al. Specificity of facial expression labeling deficits in childhood psychopathology. J Child Psychol Psychiatry. 2007 Sep; 48(9):863-871. [PubMed: 17714371]

9. McClure EB, Pope K, Hoberman AJ, Pine DS, Leibenluft E. Facial expression recognition in adolescents with mood and anxiety disorders. Am J Psychiatry. 2003 Jun; 160(6):1172-1174. [PubMed: 12777278]

10. Brotman MA, Skup M, Rich BA, et al. Risk for bipolar disorder is associated with face-processing deficits across emotions. J Am Acad Child Adolesc Psychiatry. 2008 Dec; 47(12):1455-1461.

[PubMed: 19034190] 
11. De Sonneville LMJ, Verschoor CA, Njiokiktjien C, Op het Veld V, Toorenaar N, Vranken M. Facial identity and facial emotions: Speed, accuracy, and processing strategies in children and adults. J Clin Exp Neuropsyc. 2002; 24(2):200-213.

12. Adleman NE, Kayser RR, Olsavsky AK, et al. Abnormal fusiform activation during emotional-face encoding assessed with functional magnetic resonance imaging. Psychiatry Res. 2013 May 30; 212(2):161-163. [PubMed: 23541333]

13. Brotman MA, Guyer AE, Lawson ES, et al. Facial emotion labeling deficits in children and adolescents at risk for bipolar disorder. Am J Psychiatry. 2008 Mar; 165(3):385-389. [PubMed: 18245180]

14. Kanwisher N, McDermott J, Chun MM. The fusiform face area: a module in human extrastriate cortex specialized for face perception. J Neurosci. 1997 Jun 1; 17(11):4302-4311. [PubMed: 9151747]

15. Puce A, Allison T, Asgari M, Gore JC, McCarthy G. Differential sensitivity of human visual cortex to faces, letterstrings, and textures: a functional magnetic resonance imaging study. J Neurosci. 1996 Aug 15; 16(16):5205-5215. [PubMed: 8756449]

16. Adler CM, Levine AD, DelBello MP, Strakowski SM. Changes in gray matter volume in patients with bipolar disorder. Biol Psychiatry. 2005 Jul 15; 58(2):151-157. [PubMed: 15922309]

17. Moorhead TW, McKirdy J, Sussmann JE, et al. Progressive gray matter loss in patients with bipolar disorder. Biol Psychiatry. 2007 Oct 15; 62(8):894-900. [PubMed: 17617385]

18. Allison T, Puce A, McCarthy G. Social perception from visual cues: role of the STS region. Trends Cogn Sci. 2000 Jul; 4(7):267-278. [PubMed: 10859571]

19. Perrett DI, Smith PAJ, Potter DD, et al. Visual Cells in the Temporal Cortex Sensitive to Face View and Gaze Direction. P Roy Soc B-Biol Sci. 1985; 223(1232):293-317.

20. Hoffman EA, Haxby JV. Distinct representations of eye gaze and identity in the distributed human neural system for face perception. Nat Neurosci. 2000 Jan; 3(1):80-84. [PubMed: 10607399]

21. Hasselmo ME, Rolls ET, Baylis GC, Nalwa V. Object-Centered Encoding by Face-Selective Neurons in the Cortex in the Superior Temporal Sulcus of the Monkey. Exp Brain Res. 1989; 75(2):417-429. [PubMed: 2721619]

22. Puce A, Allison T, Bentin S, Gore JC, McCarthy G. Temporal cortex activation in humans viewing eye and mouth movements. J Neurosci. 1998 Mar 15; 18(6):2188-2199. [PubMed: 9482803]

23. Pavuluri MN, O`Connor MM, Harral E, Sweeney JA. Affective Neural Circuitry During Facial Emotion Processing in Pediatric Bipolar Disorder. Biol Psychiat. 2007; 62(2):158-167. [PubMed: 17097071]

24. Pierce K, Redcay E. Fusiform function in children with an autism spectrum disorder is a matter of "who". Biol Psychiatry. 2008 Oct 1; 64(7):552-560. [PubMed: 18621359]

25. Walther S, Federspiel A, Horn H, et al. Encoding deficit during face processing within the right fusiform face area in schizophrenia. Psychiatry Res. 2009 Jun 30; 172(3):184-191. [PubMed: 19398309]

26. Pelphrey KA, Morris JP, McCarthy G, Labar KS. Perception of dynamic changes in facial affect and identity in autism. Soc Cogn Affect Neur. 2007 Jun; 2(2):140-149.

27. Findling RL, Youngstrom EA, Fristad MA, et al. Characteristics of children with elevated symptoms of mania: the Longitudinal Assessment of Manic Symptoms (LAMS) study. J Clin Psychiatry. 2010 Dec; 71(12):1664-1672. [PubMed: 21034685]

28. Horwitz SM, Demeter CA, Pagano ME, et al. Longitudinal Assessment of Manic Symptoms (LAMS) study: background, design, and initial screening results. J Clin Psychiatry. 2010 Nov; 71(11):1511-1517. [PubMed: 21034684]

29. Perlman SB. Neuroimaging in child clinical populations: considerations for a successful research program. J Am Acad Child Adolesc Psychiatry. 2012 Dec; 51(12):1232-1235. [PubMed: 23200279]

30. Weschler, D. Weschler abbreviated scale of intelligence (WASI). London: Psychological Corporation; 1999.

31. Kaufman J, Birmaher B, Brent D, et al. Schedule for affective disorders and schizophrenia for school-age children-present and lifetime version (K-SADS-PL): initial reliability and validity data. 
Journal of the American Academy of Child \& Adolescent Psychiatry. 1997; 36(7):980-988. [PubMed: 9204677]

32. Perlman SB, Almeida JR, Kronhaus DM, et al. Amygdala activity and prefrontal cortex-amygdala effective connectivity to emerging emotional faces distinguish remitted and depressed mood states in bipolar disorder. Bipolar Disord. 2012 Mar; 14(2):162-174. [PubMed: 22420592]

33. Tottenham N, Tanaka JW, Leon AC, et al. The NimStim set of facial expressions: judgments from untrained research participants. Psychiatry Res. 2009 Aug 15; 168(3):242-249. [PubMed: 19564050]

34. Keener MT, Fournier JC, Mullin BC, et al. Dissociable patterns of medial prefrontal and amygdala activity to face identity versus emotion in bipolar disorder. Psychol Med. 2012 Sep; 42(9):19131924. [PubMed: 22273442]

35. Magnotta VA, Friedman L. Measurement of signal-to-noise and contrast-to-noise in the fBIRN multicenter imaging study. Journal of Digital Imaging. 2006; 19(2):140-147. [PubMed: 16598643]

36. Segall JM, Turner JA, van Erp TGM, et al. Voxel-based morphometric multisite collaborative study on schizophrenia. Schizophrenia bulletin. 2009; 35(1):82-95. [PubMed: 18997157]

37. Talairach, JTP. Co-planar stereotaxic atlas of the human brain: 3 dimensional proportional system: An approach to cerebral imaging. New York: Thieme Medical; 1988.

38. Friston KJ, Holmes AP, Worsley KJ, Poline JP, Frith CD, Frackowiak RSJ. Statistical parametric maps in functional imaging: A general linear approach. Human Brain Mapping. 1994; 2(4):189210.

39. Lieberman MD, Cunningham WA. Type I and Type II error concerns in fMRI research: rebalancing the scale. Soc Cogn Affect Neurosci. 2009 Dec; 4(4):423-428. [PubMed: 20035017]

40. Phillips ML, Travis MJ, Fagiolini A, Kupfer DJ. Medication effects in neuroimaging studies of bipolar disorder. Am. J. Psychiatry. 2008 Mar; 165(3):313-320. [PubMed: 18245175]

41. Haxby JV, Hoffman EA, Gobbini MI. Human neural systems for face recognition and social communication. Biol Psychiatry. 2002 Jan 1; 51(1):59-67. [PubMed: 11801231]

42. George N, Driver J, Dolan RJ. Seen gaze-direction modulates fusiform activity and its coupling with other brain areas during face processing. Neuroimage. 2001 Jun; 13(6 Pt 1):1102-1112. [PubMed: 11352615]

43. Hoffman EA, Haxby JV. Distinct representations of eye gaze and identity in the distributed human neural system for face perception. Nat Neurosci. 2000 Jan; 3(1):80-84. [PubMed: 10607399]

44. Campbell R, MacSweeney M, Surguladze S, et al. Cortical substrates for the perception of face actions: an fMRI study of the specificity of activation for seen speech and for meaningless lowerface acts (gurning). Brain Res Cogn Brain Res. 2001 Oct; 12(2):233-243. [PubMed: 11587893]

45. Schultz RT. Developmental deficits in social perception in autism: the role of the amygdala and fusiform face area. Int J Dev Neurosci. 2005 Apr-May;23(2-3):125-141. [PubMed: 15749240]

46. Dalton KM, Nacewicz BM, Johnstone T, et al. Gaze fixation and the neural circuitry of face processing in autism. Nat Neurosci. 2005 Apr; 8(4):519-526. [PubMed: 15750588]

47. Kleinhans NM, Richards T, Sterling L, et al. Abnormal functional connectivity in autism spectrum disorders during face processing. Brain. 2008 Apr; 131(Pt 4):1000-1012. [PubMed: 18234695]

48. Leibenluft E, Rich BA. Pediatric bipolar disorder. Annu Rev Clin Psychol. 2008; 4:163-187. [PubMed: 17716034]

49. Pavuluri MN, O'Connor MM, Harral E, Sweeney JA. Affective neural circuitry during facial emotion processing in pediatric bipolar disorder. Biol Psychiatry. 2007 Jul 15; 62(2):158-167. [PubMed: 17097071]

50. McCarthy G, Puce A, Gore JC, Allison T. Face-specific processing in the human fusiform gyrus. J Cognitive Neurosci. 1997 Sep; 9(5):605-610.

51. Levy J, Sperry RW, Trevarth C. Perception of Bilateral Chimeric Figures Following Hemispheric Deconnection. Brain. 1972; 95:61-78. [PubMed: 5023091]

52. Halgren E, Raij T, Marinkovic K, Jousmaki V, Hari R. Cognitive response profile of the human fusiform face area as determined by MEG. Cerebral Cortex. 2000 Jan; 10(1):69-81. [PubMed: 10639397] 
53. Davidson RJ, Jackson DC, Kalin NH. Emotion, plasticity, context, and regulation: Perspectives from affective neuroscience. Psychol Bull. 2000 Nov; 126(6):890-909. [PubMed: 11107881]

54. Harmon-Jones E. Trait anger predicts relative left frontal cortical activation to anger-inducing stimuli. Int J Psychophysiol. 2007 Nov; 66(2):154-160. [PubMed: 17561297]

55. Brotman MA, Rich BA, Guyer AE, et al. Amygdala activation during emotion processing of neutral faces in children with severe mood dysregulation versus ADHD or bipolar disorder. Am J Psychiatry. 2010 Jan; 167(1):61-69. [PubMed: 19917597]

56. Pessoa L, McKenna M, Gutierrez E, Ungerleider LG. Neural processing of emotional faces requires attention. Proc Natl Acad Sci U S A. 2002 Aug 20; 99(17):11458-11463. [PubMed: 12177449]

57. Kerns JG, Cohen JD, MacDonald AW 3rd, et al. Decreased conflict- and error-related activity in the anterior cingulate cortex in subjects with schizophrenia. Am J Psychiatry. 2005 Oct; 162(10): 1833-1839. [PubMed: 16199829]

58. Botvinick MM, Braver TS, Barch DM, Carter CS, Cohen JD. Conflict monitoring and cognitive control. Psychol Rev. 2001 Jul; 108(3):624-652. [PubMed: 11488380]

59. Anderson AK, Christoff K, Panitz D, De Rosa E, Gabrieli JD. Neural correlates of the automatic processing of threat facial signals. J Neurosci. 2003 Jul 2; 23(13):5627-5633. [PubMed: 12843265]

60. Perlman SB, Hudac CM, Pegors T, Minshew NJ, Pelphrey KA. Experimental manipulation of face-evoked activity in the fusiform gyrus of individuals with autism. Soc Neurosci. 2010 May $4 .: 1-9$.

61. Bolte S, Hubl D, Feineis-Matthews S, Prvulovic D, Dierks T, Poustka F. Facial affect recognition training in autism: can we animate the fusiform gyrus? Behav Neurosci. 2006 Feb; 120(1):211216. [PubMed: 16492133] 


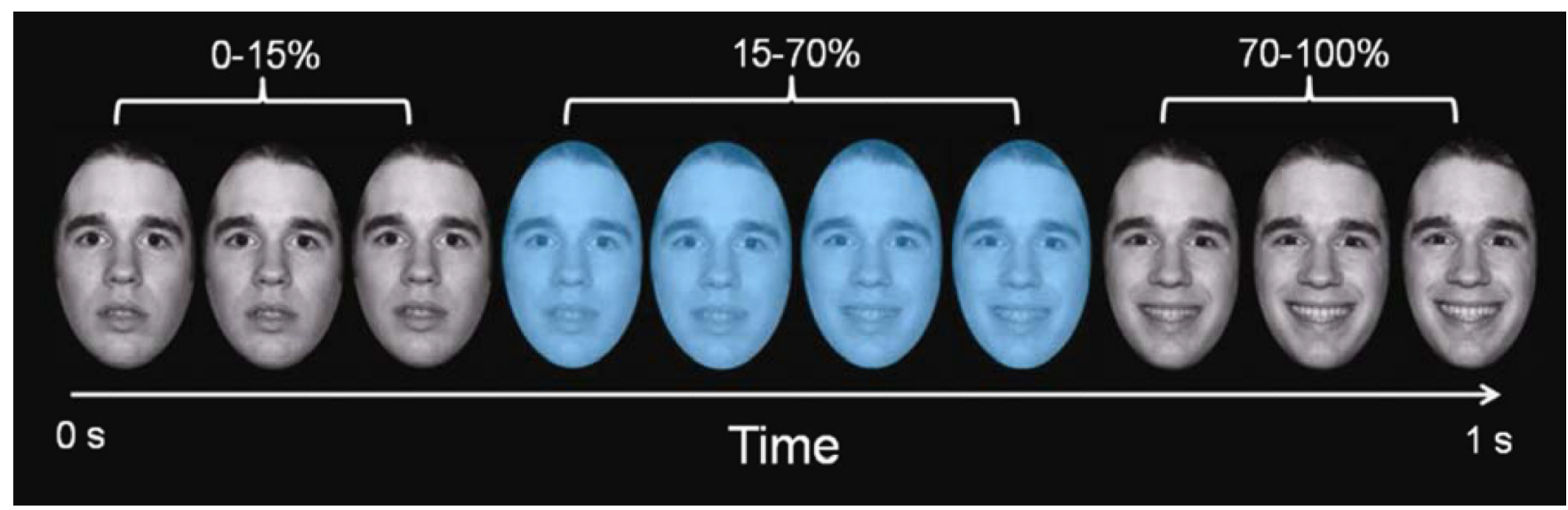

Figure 1.

Dynamic Faces Task. Note: Graphic representation of a single happy trial of our emotional dynamic faces task. Over a one-second duration, the face changed from neutral $(0 \%$ emotion) to a happy, sad, angry, or fearful face (100\% emotion). Participants were asked to identify the color flash presented in mid dynamic change. 


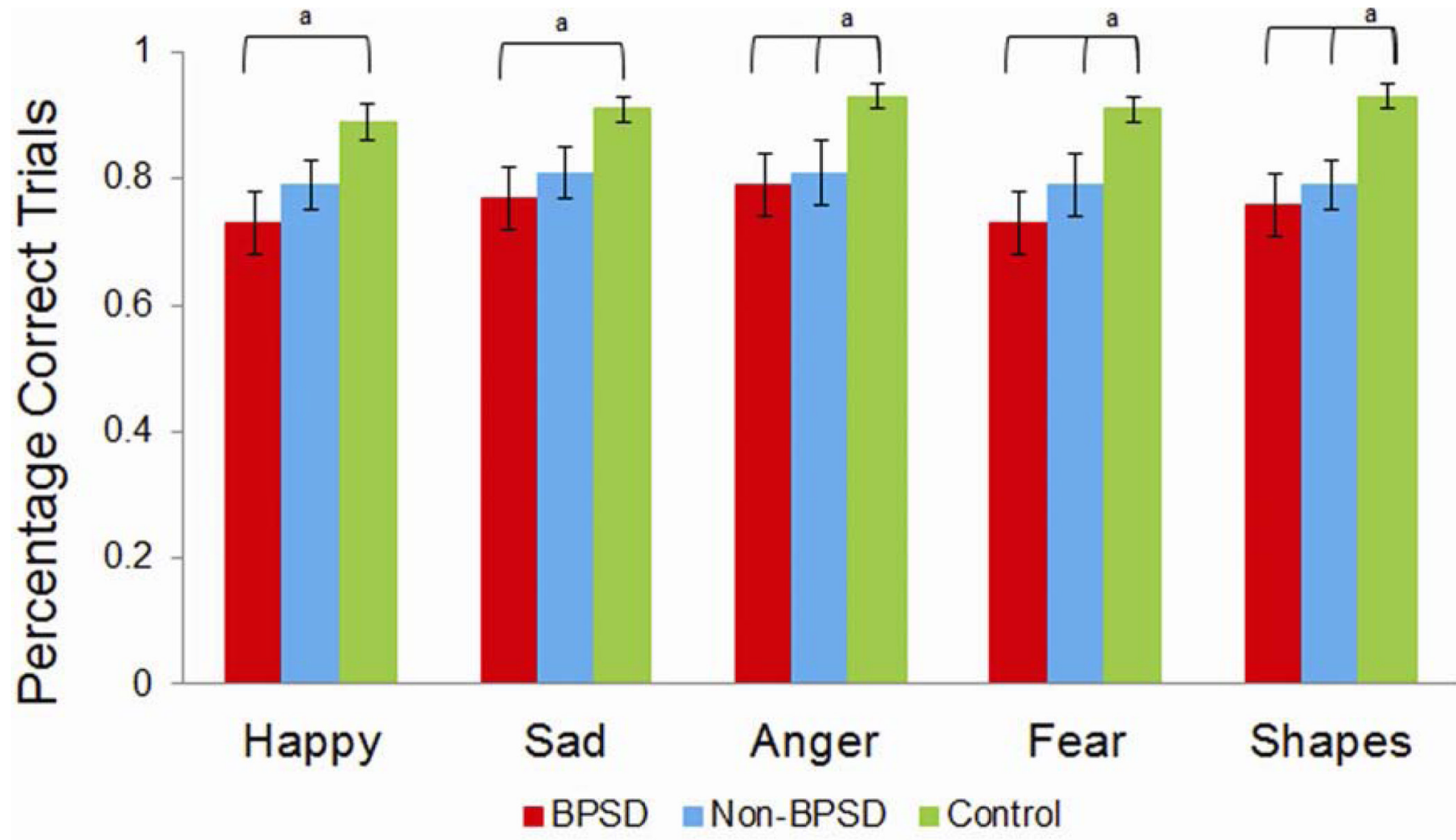

Figure 2.

Task Accuracy. Note: Task accuracy for each group and condition. The bipolar spectrum disorder (BPSD) group and the Non-BPSD group did not significantly differ from each other

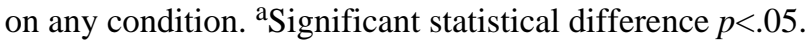



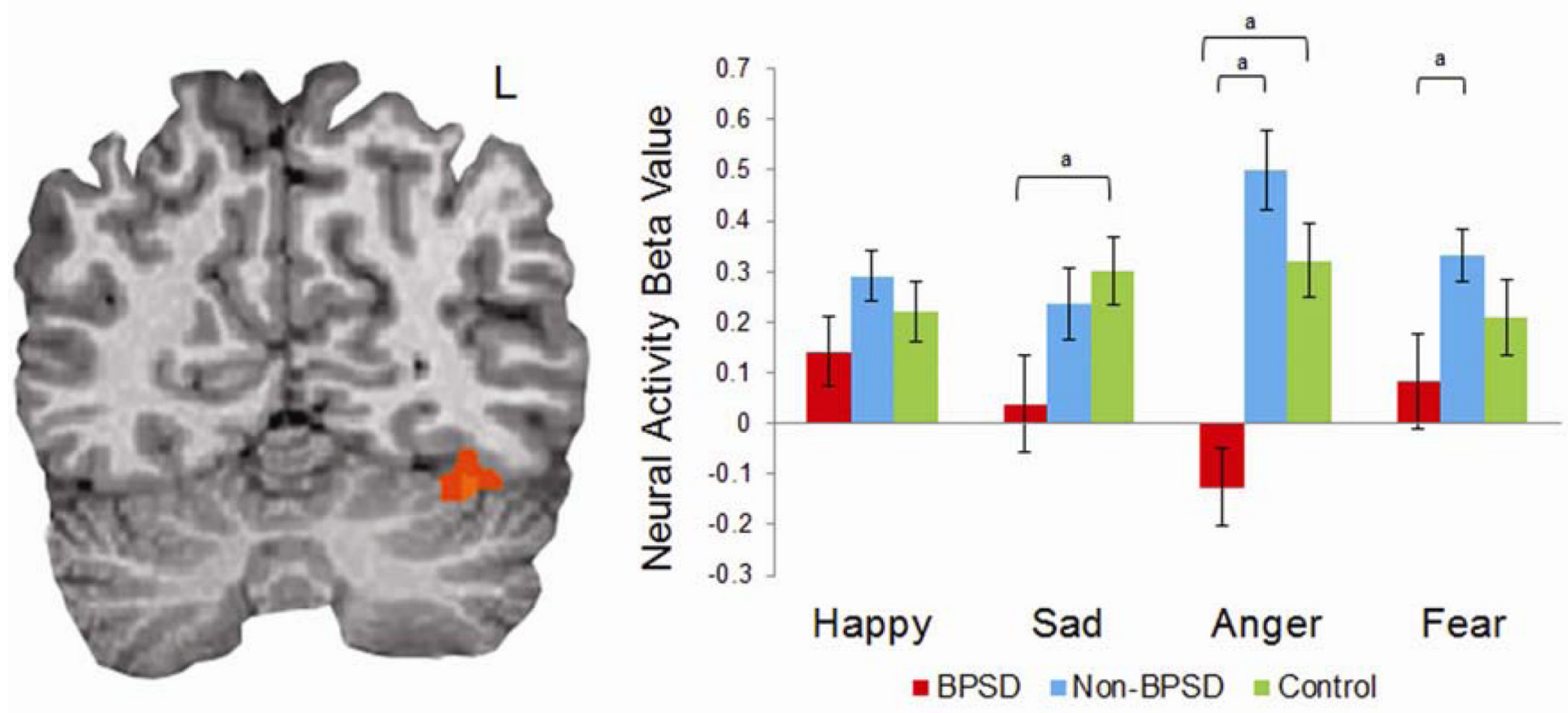

Figure 3.

Group $\times$ Condition Interaction. Note: Results of a whole-brain group $\times$ condition interaction, $(F[6,171] \geq 3.95, p<.001)$, show decreased activity in the left fusiform gyrus indicated by red highlights (TAL peak: $\mathrm{x}=-40, \mathrm{y}=-65, \mathrm{z}=-15$ ). The bipolar spectrum disorder (BPSD) group displayed significantly less activity in this region than both the Non-BPSD and Control

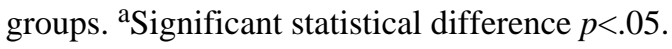




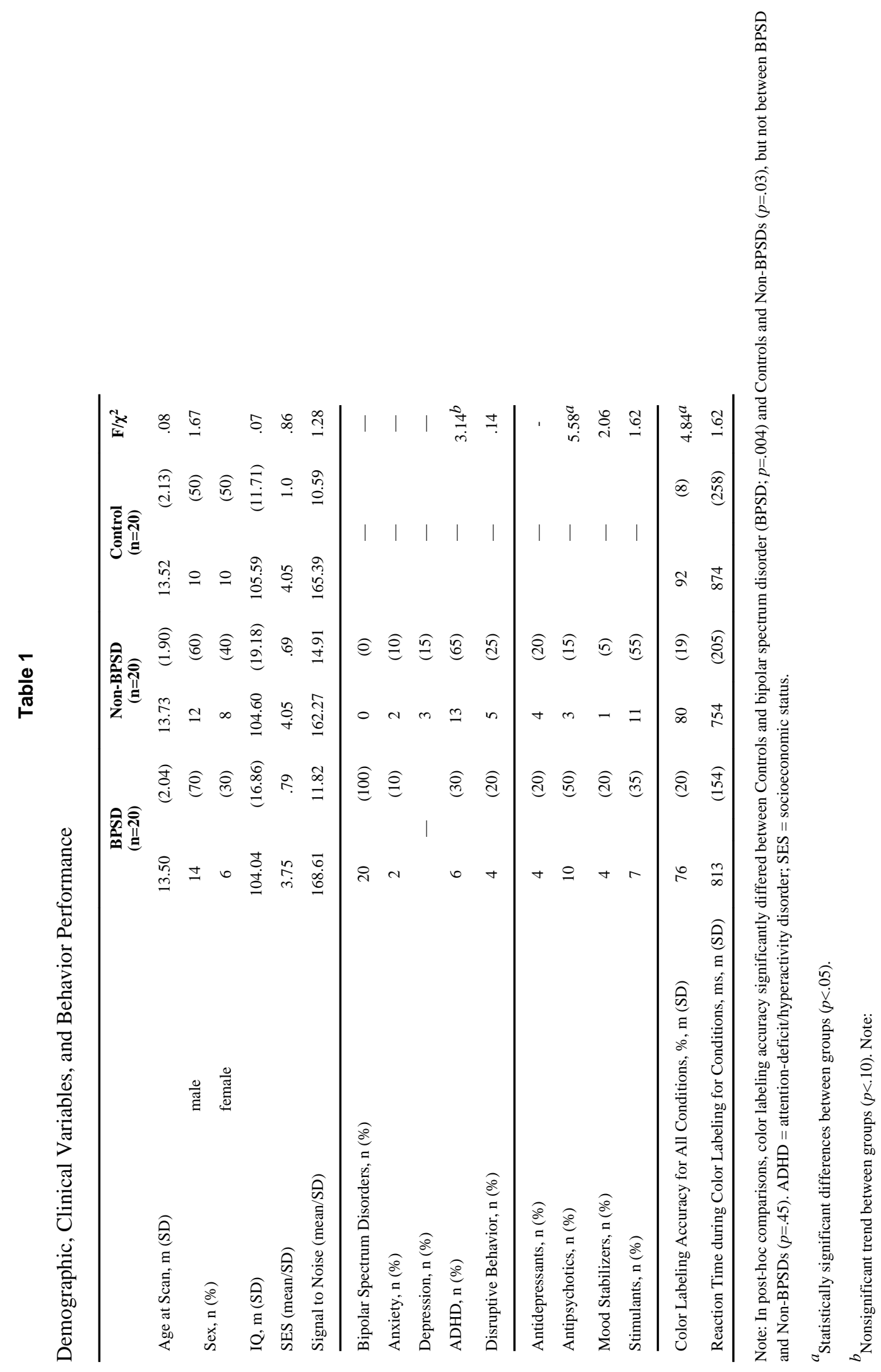




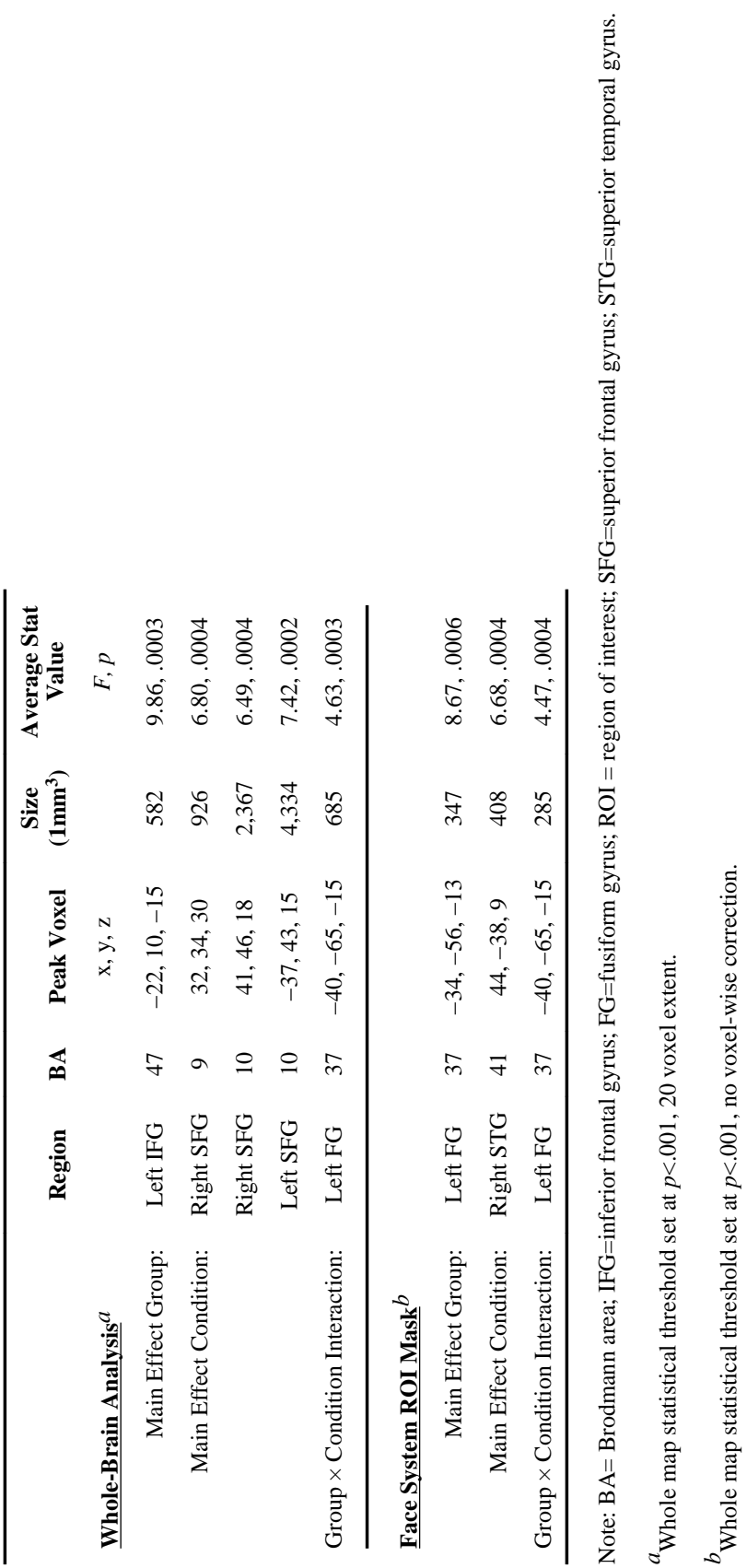

